Brown's method-each player having 5 strategies. The method provides convergence to the value of the game within .01 after about 8 hours of computing (1000 iterations) for the games computed.

3) Solution of $n$ simultaneous linear equations by Crout's method was performed for $n=5$, and a general program was prepared for $n \leqslant 10$.

4) The Project used the computer to assist in obtaining a research solution to a fleet logistics planning problem.

5) Currently, a calculation is being performed which obtains information pertaining to feasible schedules for tanker delivery of fuel. Later this information will be used to obtain an optimal schedule with the hope of application to monthly delivery of petroleum products by the Military Sea Transport Service.

The George Washington University

J. JAY WoLF

Washington, D. C.

This work was sponsored by the Office of Naval Research.

1 Publications available from the Logistics Research Project are: Jos. B. KRUSKAL, JR., A Programmer's Description of ABEL, A Magnetic Drum Relay Computer, Orders for the ONR Relay Computer, a detailed description of the instructions, and ONR Relay Computer Operators Manual.

\title{
On the Rounding Off of Difference Tables for Linear Interpolation
}

In order to simplify linear interpolation many tables contain the differences $\Delta(x)=f(x+d)-f(x)$ between two consecutive values of the function. Since the values of $f(x)$ given in the tables are usually rounded off the question arises whether the value of $\Delta(x)$ given in the table must be the difference between the rounded off values $\overline{f(x)}$ of $f(x)$ and $\overline{f(x+d)}$ of $f(x+d)$ or $\bar{\Delta}$, the result of the rounding off applied to $\Delta(x)$. It appears on the first view plausible that we obtain better results in the second case since we use here more information about $f(x)$. However, the detailed analysis shows that this is not so. If the values $f(x)$ are given with $n$ decimals so that the rounding off errors do not exceed $h=\frac{1}{2} 10^{-n}$, the part of the interpolation error due to the rounding off of $f(x)$ and $f(x+d)$ does not exceed $h$, if the difference used is $\overline{f(x+d)}-\overline{f(x)}$, while if we use $\bar{\Delta}$, this error can come arbitrarily near to $2 h$.

Since this situation is not apparently realized by all computers of tables, I should like to develop an observation on this subject which was published elsewhere. ${ }^{1}$

We give first an example.

In computing the decimal logarithm log 9684.8 we start from the values $\log 9684=3.98605478 ; \log 9685=3.98609963$ and from the rounded off values $\log 9684=3.98605 ; \log 9685=3.98610$ with an error $<h=\frac{1}{2} 10^{-5}$.

We have

$$
\Delta=4.485 \cdot 10^{-5}, \quad \bar{\Delta}=4 \cdot 10^{-5} .
$$

We obtain then by the "complete" interpolation $\log 9684.8=3.98605478+0.8 \cdot 4.485 \cdot 10^{-5}=3.98609066$, 
while in using the rounded off values of both logarithms and $\bar{\Delta}$ we obtain

$$
3.98605+0.8 \cdot 4 \cdot 10^{-5}=3.986082 \text {. }
$$

The difference is $.866 \cdot 10^{-5}>h$, while, if we take instead of $\bar{\Delta}$ the difference $5 \cdot 10^{-5}$ of the rounded off values, we obtain the value 3.98609 and the whole rounding off error is $.066 \cdot 10^{-5}<h$.

From the following theoretical discussion it follows in particular, that if the rounding off errors of $f(x)$ and $f(x+d)$ can be considered as independent random variables, the probability that the rounding off error of the value of $f(x+t d)$, obtained in using $\bar{\Delta}$, is $>(1+\eta) h, 0 \leqq \eta<1$, is equal to $\frac{1}{4}\left(t-2 \eta+\eta^{2} / t\right)$, if $1>t>\eta$. Without loss of generality, we can consider a function $f(x)$ with the two values $f(0)=f_{0}, f(1)=f_{1}$. Then for $0<t<1$, linear interpolation gives

$$
L(t)=(1-t) f_{0}+t f_{1}=f_{0}+t \Delta, \quad \Delta=f_{1}-f_{0} .
$$

For an $h=\frac{1}{2} 10^{-n}$ we denote generally by $\bar{a}$ the result of rounding off of $a$ to $n$ decimals, so that we have generally

$$
|a-\bar{a}| \leqq h, \quad \bar{a} \equiv 0(\bmod 2 h) .
$$

We use the notations

$$
\begin{gathered}
f_{i}=\bar{f}_{i}+\epsilon_{i}, \quad\left|\epsilon_{i}\right| \leqq h, \quad(i=0,1), \\
\bar{\Delta}=\overline{f_{1}-f_{0} .}
\end{gathered}
$$

With $\bar{\Delta}$ we form

$$
\begin{gathered}
L^{*}=\bar{f}_{0}+t \bar{\Delta} \\
L-L^{*}=\epsilon_{0}+t(\Delta-\bar{\Delta}) .
\end{gathered}
$$

We consider now for a constant $\eta$ with $0 \leqq \eta<1$ in the $\epsilon_{0}$, $\epsilon_{1}$-plane the set $S(t, \eta)$ of points for which we have

that is,

$$
\left|L-L^{*}\right|>(1+\eta) h
$$

$$
\left|\epsilon_{0}+t(\Delta-\bar{\Delta})\right|>(1+\eta) h
$$

where

$$
\left\{\begin{array}{c}
\Delta-\bar{\Delta} \equiv \epsilon_{1}-\epsilon_{0}(\bmod 2 h), \\
|\Delta-\bar{\Delta}|<h .
\end{array}\right.
$$

Our problem is to determine the area of $S(t, \eta)$. The inequality (7) can be satisfied only for $t>\eta$, since $\left|\epsilon_{0}\right| \leqq h,|\Delta-\bar{\Delta}| \leqq h$.

$$
\left|\epsilon_{0}\right| \leqq h, \quad\left|\epsilon_{1}\right| \leqq h .
$$

Further we must have

$$
\left|\epsilon_{1}-\epsilon_{0}\right|>h,
$$

since otherwise $\bar{\Delta}$ would be $\bar{f}_{1}-\bar{f}_{0}$ and in that case the left side of (7) is $\leqq h$.

We will have therefore two cases accordingly as

$$
\text { I. } \epsilon_{1}-\epsilon_{0}>h \text { or II. } \epsilon_{1}-\epsilon_{0}<-h \text {. }
$$


In both cases we have

$$
\epsilon_{1} \epsilon_{0}<0 .
$$

Case $I . \quad h \geqq \epsilon_{1}>0>\epsilon_{0} \geqq-h, \epsilon_{1}-\epsilon_{0}>h$.

Here we have from (8)

and (7) becomes

$$
\Delta-\bar{\Delta}=\epsilon_{1}-\epsilon_{0}-2 h,
$$

$$
\epsilon_{0}+t\left(\epsilon_{1}-\epsilon_{0}\right)-2 t h<-(1+\eta) h,
$$

since the left hand expression in (11) cannot be $>h$.

The points satisfying (11) lie beneath the straight line

$$
(1-t) \epsilon_{0}+t \epsilon_{1}+(1+\eta-2 t) h=0 .
$$

The line (12) meets $\epsilon_{0}=-h$ in the point

$$
P_{0}\left(\epsilon_{0}=-h, \epsilon_{1}=(1-\eta / t) h\right)
$$

and the line $\epsilon_{1}-\epsilon_{0}=h$ in the point

$$
P_{1}\left(\epsilon_{0}=(t-\eta-1) h, \epsilon_{1}=(t-\eta) h\right) .
$$

The corresponding part of the set $S(t, \eta)$ is therefore the shaded,triangle in the diagram

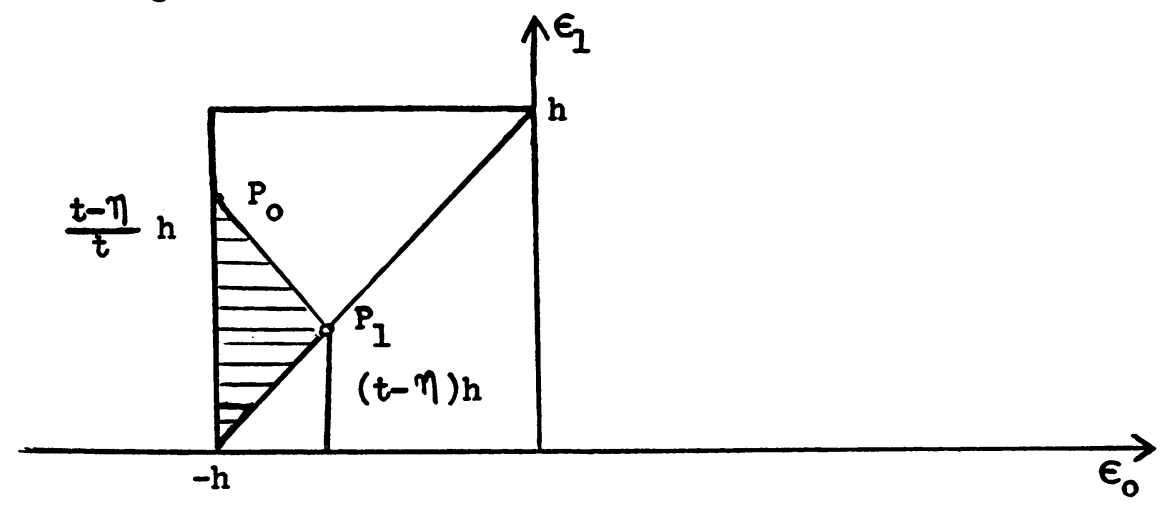

and has the area $\frac{1}{2}(t-\eta)^{2} h^{2} / t$. In the case II we have an entirely symmetric argument and have therefore for the total area of $S(t, \eta)$ the expression $(t-\eta)^{2} h^{2} / t$, while all points $\left(\epsilon_{0}, \epsilon_{1}\right)$ satisfying (9) cover the area $4 h^{2}$.

We see that the "geometric" probability that the point $\left(\epsilon_{0}, \epsilon_{1}\right)$ will fall into the set $S(t, \eta)$ is $\frac{1}{4}(t-\eta)^{2} / t$, an expression that is not only positive for $t>\eta$, but even fairly large.

American University

A. M. Ostrowski

Washington, D. C.

This work was performed on a National Bureau of Standards contract with the American University.

1 A. Ostrowski, Vorlesungen über Differential und Integralrechnung. Basel, 1951, v. 2, p. 294-296. 